\title{
Progressive dysphagia and neck pain due to diffuse idiopathic skeletal hyperostosis of the cervical spine: a case report and literature review
}

This article was published in the following Dove Press journal:

Clinical Interventions in Aging

3I March 2014

Number of times this article has been viewed

\section{Chao Zhang \\ Dike Ruan \\ Qing $\mathrm{He}$ \\ Tianyong Wen \\ Pushan Yang}

Department of Orthopedic Surgery,

Navy General Hospital, Beijing,

People's Republic of China
Correspondence: Chao Zhang

Department of Orthopedic Surgery, Navy General Hospital, Beijing Fucheng 6\#

100048, People's Republic of China

Tel +861068780323

Fax +86 1068780323

Email zhangchaongh@।63.com

\begin{abstract}
Diffuse idiopathic skeletal hyperostosis (DISH) is considered an underdiagnosed and mostly asymptomatic nonprimary osteoarthritis. The etiology of DISH remains unknown and the validated diagnostic criteria are absent. This condition is still recognized radiologically only. Rarely, large projecting anterior osteophytes result in esophageal impingement and distortion leading to dysphagia. We report the case of progressive dysphagia and neck pain due to DISH of the cervical spine in a 70-year-old man, which was surgically removed with excellent postoperative results and complete resolution of symptoms. Imaging studies, surgical findings, and histopathological examinations were used to support the diagnosis. The patient was successfully treated with total excision of the anterior osteophytes with no evidence of recurrence 12 months after surgery. In this report, we also discuss the clinical features and perioperative considerations in combination with a literature review. Our patient illustrates that clinicians should be aware of this rare clinical manifestation as the presenting feature of DISH in cervical spine. Surgical decompression through osteophytectomy is effective for patients who fail conservative treatment.
\end{abstract}

Keywords: diffuse idiopathic skeletal hyperostosis, cervical spine, dysphagia

\section{Introduction}

Diffuse idiopathic skeletal hyperostosis (DISH), also known as Forestier's disease, was first described by Forestier and Rotes-Querol in $1950 .{ }^{1}$ It is characterized radiologically by flowing calcification along the sides of the contiguous vertebrae of the spine. This ectopic calcification can lead to limitation of motion of the involved areas of the spine, which causes stiffness and dull pain. DISH is slowly progressive and the pain is usually intermittent and thus overlooked and neglected by patients and physicians. Rarely, large bone spurs can form in front of the cervical vertebrae. These spurs occasionally interfere with the passage of food through the esophagus. We present a case report of a male patient seen in the orthopedic clinic with chronic neck pain and dysphagia secondary to DISH and present a review of the literature.

\section{Case report}

The study has been approved by the Committee for Human Research at Navy General Hospital, and written informed consent was collected from the patient.

A 71-year-old Asian man was referred to the orthopedic clinic of Navy General Hospital with the main complaint of chronic neck pain for 5 years and increased difficulty in swallowing solid food over the past 3 years. The neck pain was dull and progressive but generally tolerated by the patient. Sometimes neck pain would become sharp with certain body movements such as twisting or bending over. Over the past 
year he had altered his diet to include only soft foods and liquids, and he had an approximately $5 \mathrm{~kg}$ weight loss over that period of time.

Esophagogastroduodenoscopy had been done 2 years before, and the results were unremarkable. He denied difficulty in breathing and any symptoms suggestive of cervical radiculopathy or myelopathy. The patient had had type 2 diabetes for 12 years and had been admitted to the local hospital on two previous occasions for control of his disease.

On physical examination, the patient was alert, afebrile, and well oriented, with stable vital signs. Cervical range of motion was significantly decreased, and a hard lump could be felt deep in the anterolateral cervical region. The neurological exam and the patient's reflexes were normal. The patient did not show clinical, radiological, or serological evidence of rheumatoid arthritis or of ankylosing spondylitis. His neck disability index score was $62 \%$ and the visual analogue scale score for neck pain was 7 while hospitalized in our clinic.

Laboratory tests including blood cell count, C-reactive protein, erythrocyte sedimentation rate, and autoimmune panel were normal. The lateral radiograph of his cervical spine showed characteristic flowing ossification along the anterior aspect of the cervical vertebrae from $\mathrm{C} 2-\mathrm{C} 5$, with relative preservation of the disc spaces (Figure 1). The facet joints

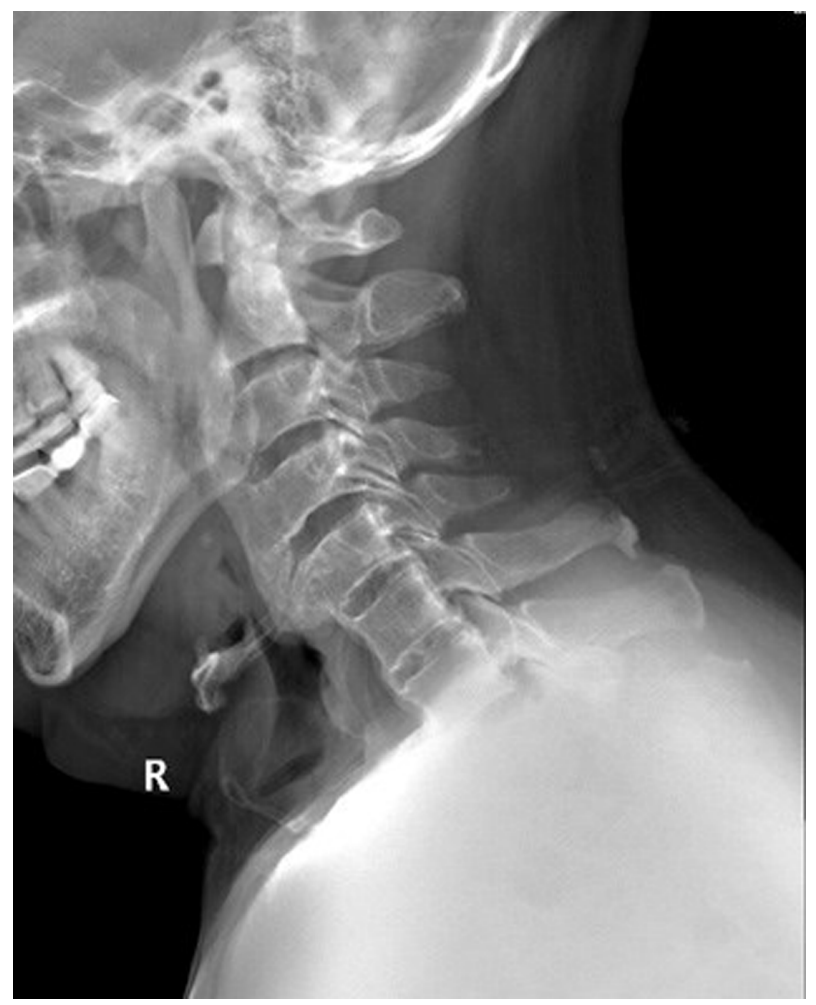

Figure I The lateral radiograph of cervical spine shows characteristic flowing ossification along the anterior aspect of the cervical vertebrae from C2-C5. and spinous processes did not appear ankylosed. The bony mass compressed the esophagus anteriorly and resulted in marked swallowing tube stenosis. The computed tomography scan of the cervical spine revealed continuous but irregular flowing hyperostosis alongside the anterior aspect of all the cervical vertebrae as well as local ossification of the posterior longitudinal ligament (Figure 2). There were especially striking osteophyte outgrowths covering the anterior side of the vertebrae and projecting into the soft tissues of the neck from the level of C2 to C5 (Figure 3). The largest bony excrescences measured $16.32 \mathrm{~mm}$ thick. The anterior edge of the body of the vertebrae was clearly visible at the posterior limit of the outgrowth except at C4. Sagittal T2-weighted magnetic resonance imaging showed bone marrow signal abnormality (high signal intensity) in C5-C7 as well as in the outgrowth hyperostosis (Figure 4). Magnetic resonance imaging did not show obvious spinal stenosis and cord compression. The features were characteristic of DISH or Forestier's disease.

\section{Results}

Conservative therapy was insufficient for this patient, therefore, we performed osteophytectomy. Difficult intubation was anticipated in view of the radiological findings and restricted mobility of the neck. We discussed anesthetic choices and intubating techniques with the anesthetist preoperatively. The patient was placed under general anesthesia with extreme caution to not over rotate or extend his neck. He underwent operative excision of the anterior osteophytes through routine anterolateral approach to the cervical spine. On histologic examination, the appearance of trabecular

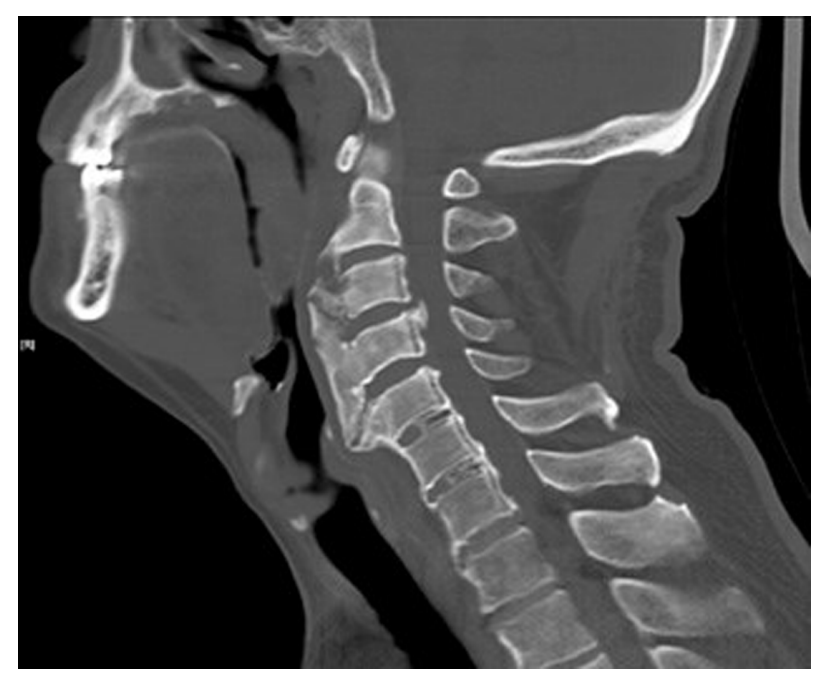

Figure 2 The preoperative CT scan of the cervical spine reveals continuous but irregular flowing hyperostosis alongside the anterior aspect of the whole cervical vertebrae.

Abbreviation: $\mathrm{CT}$, computed tomography. 


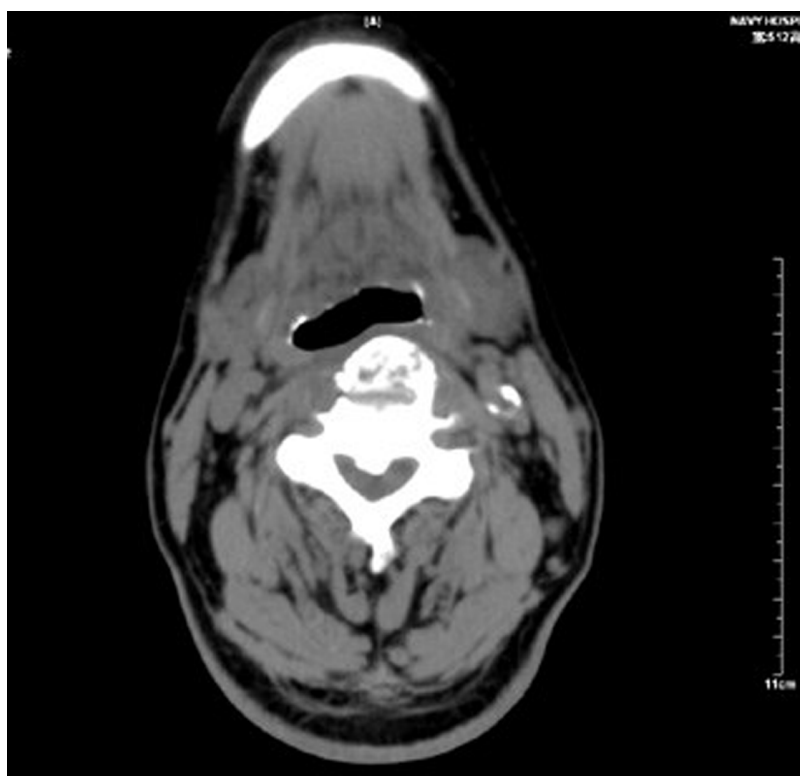

Figure 3 The axial CT scan shows outgrowth osteophytes covering the anterior side of the vertebrae as well as local ossification of the posterior longitudinal ligament.

Abbreviation: CT, computed tomography.

bone in the fibrous tissue with spindle cell proliferation was noted. Primitive cartilage and immature woven bone could also be seen. The resected sample also showed nonspecific diffuse chronic inflammation in the trabecular bone and connective tissue infiltrated with a mixed population of lymphocytes, plasma cells, and macrophages (Figure 5). However,

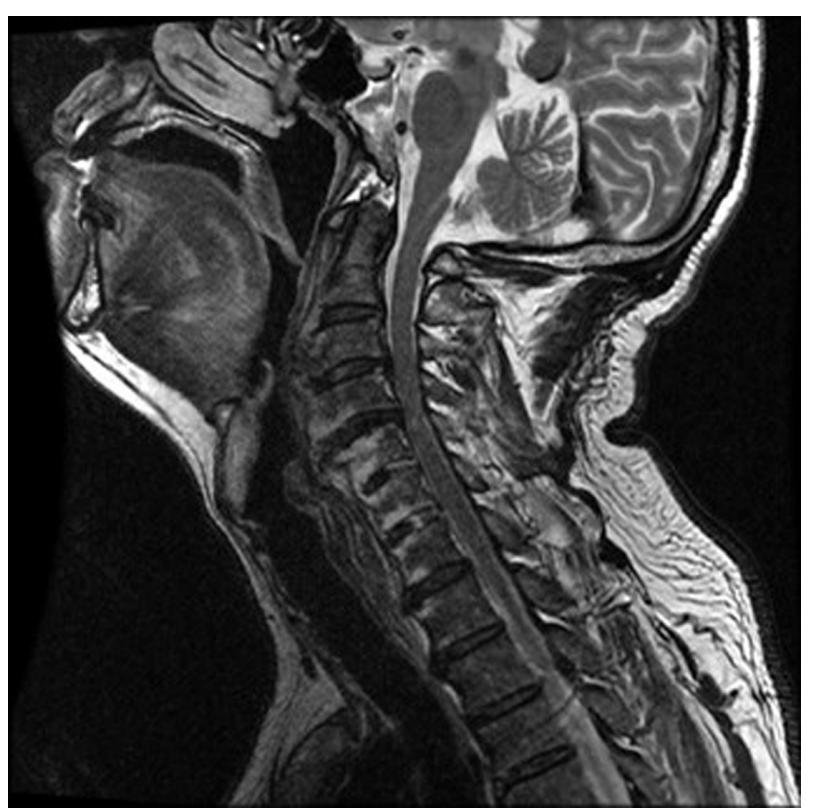

Figure 4 The preoperative T2-weighted sagittal magnetic resonance spin-echo image shows high signal intensity of bone marrow in C5-C7 as well as in the outgrowth hyperostosis.

Note: MRI did not show obvious spinal stenosis and cord compression. Abbreviation: MRI, magnetic resonance imaging.

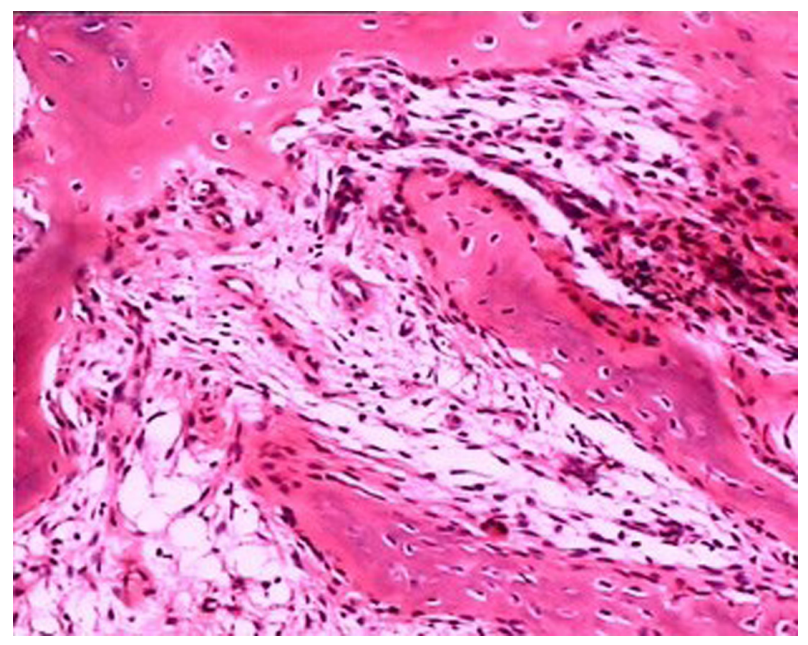

Figure 5 Histologic appearance of the removed tissue shows trabecular bone in the fibrous tissue with spindle cell proliferation.

Notes: The resected sample also shows nonspecific diffuse chronic inflammation in the trabecular bone and connective tissue, infiltrated with a mixed population of lymphocytes, plasma cells, and macrophages. Hematoxylin and eosin $(\times 100)$.

the general pattern of distribution of these changes was quite distinct from, and of lower intensity than, that of chronic inflammatory arthritis such as ankylosing spondylitis. The postoperative radiological studies confirmed that the massive ectopic osteophytes had been removed, and the structure of prevertebral tissues was anatomically normal (Figure 6). The patient had residual dysphagia in the first 2 weeks following surgery due to traction of the trachea and esophagus but this gradually improved. At the time of his most recent follow-up, 12 months after the surgery, the patient had marked improvement in swallowing function and was able to resume a normal diet. His neck disability index score improved from $62 \%$ preoperatively to $28 \%$ postoperatively, and his visual analogue scale score for neck pain improved from 7 to 2 . The patient provided his consent for the publication of this case report.

\section{Discussion}

DISH is a noninflammatory condition characterized by calcification and ossification of soft tissues, mainly ligaments and enthesis. Although it is considered a form of degenerative arthritis or osteoarthritis, DISH is also commonly associated with inflammation and calcification of tendons or ligaments at their attachment points to bone, especially in the region of the spine. The ossification and calcification of these prevertebral structures is responsible for the characteristic radiographic appearance. A characteristic radiographic feature of spine involvement is the presence of hyperostosis of the cortex along the anterior surface of the vertebrae. Gradually, elongated osteophytes occur at the anterior margin of the vertebrae 


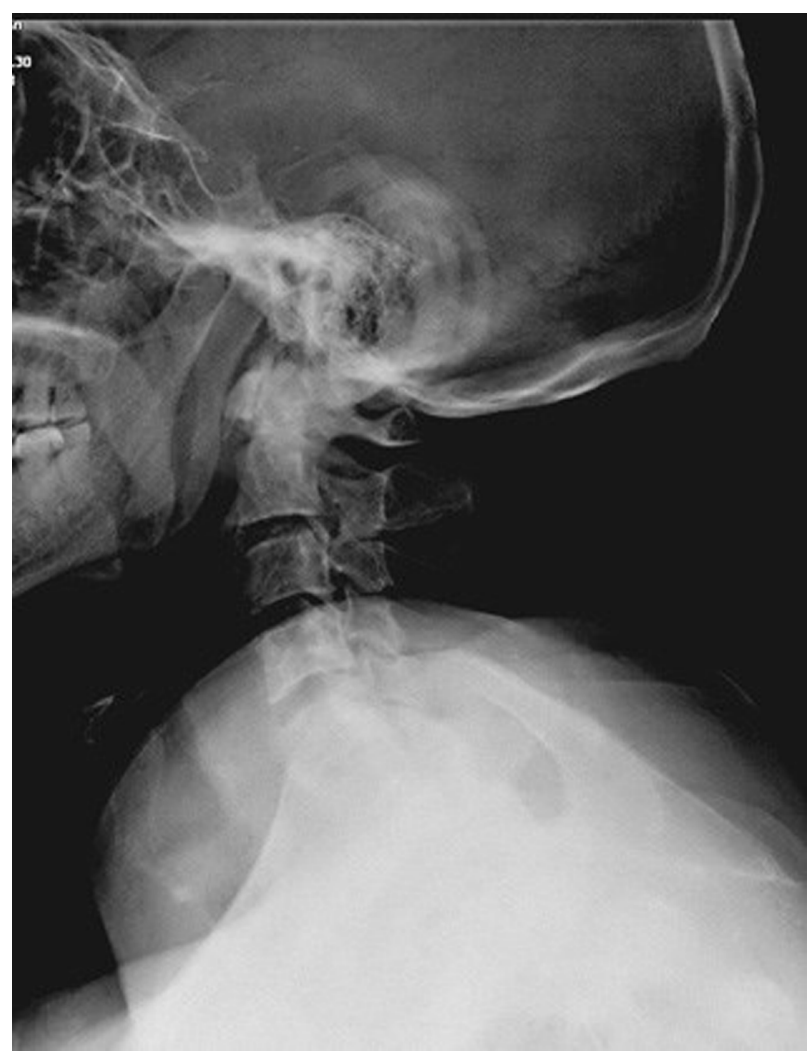

Figure 6 Postoperative lateral radiograph of cervical spine showing the anatomically normal structure of prevertebral tissues.

and grow across the disc space. ${ }^{2,3}$ In most textbooks, DISH is described as "asymptomatic", and it is often accidentally found by radiographs of the spine performed for restriction in movement of spinal joints and enthesis-related pain. ${ }^{4,5}$ Whether the condition itself is a cause of significant pain and whether it is a true disease entity still remain controversial. ${ }^{6}$

The exact mechanism of DISH remains speculative; various hypotheses include mechanical factors, diet, metabolic conditions, and environmental exposures. ${ }^{7}$ Clinical observations demonstrate that the condition is associated with the male sex, obesity, diabetes, and advanced age. In addition, DISH is probably related to abnormal bone cell growth activity, reflecting the influence of metabolic factors that lead to new bone formation. Serum matrix Gla protein may be a marker of osteometabolic syndromes, such as DISH, that cause hyperostosis. ${ }^{8}$ Histological findings from this patient indicated obvious inflammatory reaction in the ossification tissues, which suggests that nonspecific inflammation might also play an important role in the etiology of DISH, although it is usually considered a noninflammatory disease. The histological features of inflammatory response within these layers of the new bone formation have never been previously detailed and reviewed.
Currently, DISH is still recognized radiologically, and there are several criteria that could be considered to establish diagnosis. Julkunen's criteria describes bony bridges between two vertebral bodies, and at least two sites of the spine must be involved to be characterized as DISH. ${ }^{9}$ Resnick and Niwayama set criteria to define DISH that require involvement of at least four contiguous vertebrae, preservation of the intervertebral disc space, and absence of apophyseal joints or sacroiliac inflammatory changes. ${ }^{10}$ Utsinger proposed criteria requiring three contiguous vertebral bodies but added the presence of extraspinal ossification. ${ }^{11}$

Although the cervical spine is much more mobile than the other parts of the spine, DISH occurs in the cervical region much less frequently than in the thoracic or lumbar regions of the spine. The process of slowly progressive DISH often causes no serious symptoms, however, localization and involvement of adjacent tissues of DISH can lead to a variety of clinical consequences. Unlike "occult" DISH of thoracic and lumbar spine, prominent cervical osteophytes occasionally cause dysphagia and/or airway obstruction. Surgical treatment is usually indicated in these rare conditions. We performed simple resection of the C2-C5 DISH segments for this patient, and the main consideration was whether a fusion procedure should be attempted postoperatively for spinal stability. Massive anterior osteophytes result in airway impingement and distortion leading to difficulty during intubation. DISH can be complicated by cervical spinal fracture resulting from even minor trauma such as over extension. Great care must be taken, and awake intubation may be another option.

\section{Conclusion}

In summary, DISH may become more widespread due to its association with age, obesity, and type 2 diabetes mellitus while its definitive natural history and diagnostic criteria remain controversial. Given the fact that degenerative spurring is usually not taken into account for the diagnosis of DISH in spine clinics, the condition may be not so uncommon and asymptomatic as we thought. This unrecognized condition may provide a new challenge for spine surgeons who are not as familiar with DISH as rheumatologists. Our patient illustrates that clinicians should be aware of this rare clinical manifestation as the presenting feature of DISH in the cervical spine. Surgical decompression through osteophytectomy is effective for patients who fail conservative treatment.

\section{Disclosure}

The authors report no conflicts of interest in this work. 


\section{References}

1. Forestier J, Rotes-Querol J. Senile ankylosing hyperostosis of the spine. Ann Rheum Dis. 1950;9(4):321-330.

2. Giuffra V, Giusiani S, Fornaciari A, Villari N, Vitiello A, Fornaciari G. Diffuse idiopathic skeletal hyperostosis in the Medici, Grand Dukes of Florence (XVI century). Eur Spine J. 2010;19(Supp1 2):S103-S107.

3. Mader R, Verlaan JJ, Buskila D. Diffuse idiopathic skeletal hyperostosis: clinical features and pathogenic mechanisms. Nat Rev Rheumatol. 2013;9(12):741-750.

4. Childs SG. Diffuse idiopathic skeletal hyperostosis: Forestier's disease. Orthop Nurs. 2004;23(6):375-382.

5. Presutti L, Alicandri-Ciufelli M, Piccinini A, et al. Forestier disease: single-center surgical experience and brief literature review. Ann Otol Rhinol Laryngol. 2010;119(9):602-608.

6. Hutton C. DISH: a state not a disease? Br J Rheumatol. 1989;28(4): $277-278$.
7. Ohishi H, Furukawa K, Iwasaki K, et al. Role of prostaglandin I 2 in the gene expression induced by mechanical stress in spinal ligament cells derived from patients with ossification of the posterior longitudinal ligament. J Pharmacol Exp Ther. 2003;305(3):818-824.

8. Sarzi-Puttini P, Atzeni F. New developments in our understanding of DISH (diffuse idiopathic skeletal hyperostosis). Curr Opin Rheumatol. 2004;16(3):287-292.

9. Julkunen H, Heinonen OP, Knekt P, Maatela J. The epidemiology of hyperostosis of the spine together with its symptoms and related mortality in a general population. Scand J Rheumatol. 1975;4(1):23-27.

10. Resnick D, Niwayama G. Diffuse idiopathic skeletal hyperostosis (DISH): ankylosing hyperostosis of Forestier and Rotes-Querol. Diagnosis of Bone and Joint Disorders. 2nd ed. Philadelphia, PA: WB Saunders 1988:1563-1615.

11. Utsinger PD. Diffuse idiopathic skeletal hyperostosis. Clin Rheum Dis. 1985;11(2):325-351.
Clinical Interventions in Aging

\section{Publish your work in this journal}

Clinical Interventions in Aging is an international, peer-reviewed journal focusing on evidence-based reports on the value or lack thereof of treatments intended to prevent or delay the onset of maladaptive correlates of aging in human beings. This journal is indexed on PubMed Central, MedLine, the American Chemical Society's 'Chemical Abstracts Ser-

\section{Dovepress}

vice' (CAS), Scopus and the Elsevier Bibliographic databases. The manuscript management system is completely online and includes a very quick and fair peer-review system, which is all easy to use. Visit http://www.dovepress.com/testimonials.php to read real quotes from published authors. 